\title{
Observations initiales et finales de Filles eveillées : Programme pilote de renforcement des compétences des adolescentes migrantes employées de maison. Cohorte 1 (2011-2012), Bobo- Dioulasso
}

Sarah Engebretsen

Population Council

Follow this and additional works at: https://knowledgecommons.popcouncil.org/departments_sbsr-pgy

Part of the Demography, Population, and Ecology Commons, Family, Life Course, and Society Commons, Gender and Sexuality Commons, International Public Health Commons, Medicine and Health Commons, and the Women's Health Commons How does access to this work benefit you? Let us know!

\section{Recommended Citation}

Engebretsen, Sarah. 2012. "Observations initiales et finales de Filles eveillées : Programme pilote de renforcement des compétences des adolescentes migrantes employées de maison. Cohorte 1 (2011-2012), Bobo-Dioulasso." Ouagadougou: Population Council. 
OBSERVATIONS INITIALES ET FINALES DE FILLES EVEILLEEES :

PROGRAMME PILOTE DE RENFORCEMENT

DES COMPÉTENCES DES ADOLESCENTES

MIGRANTES EMPLOYÉES DE MAISON

COHORTE 1 (2011-2012), BOBO-DIOULASSO

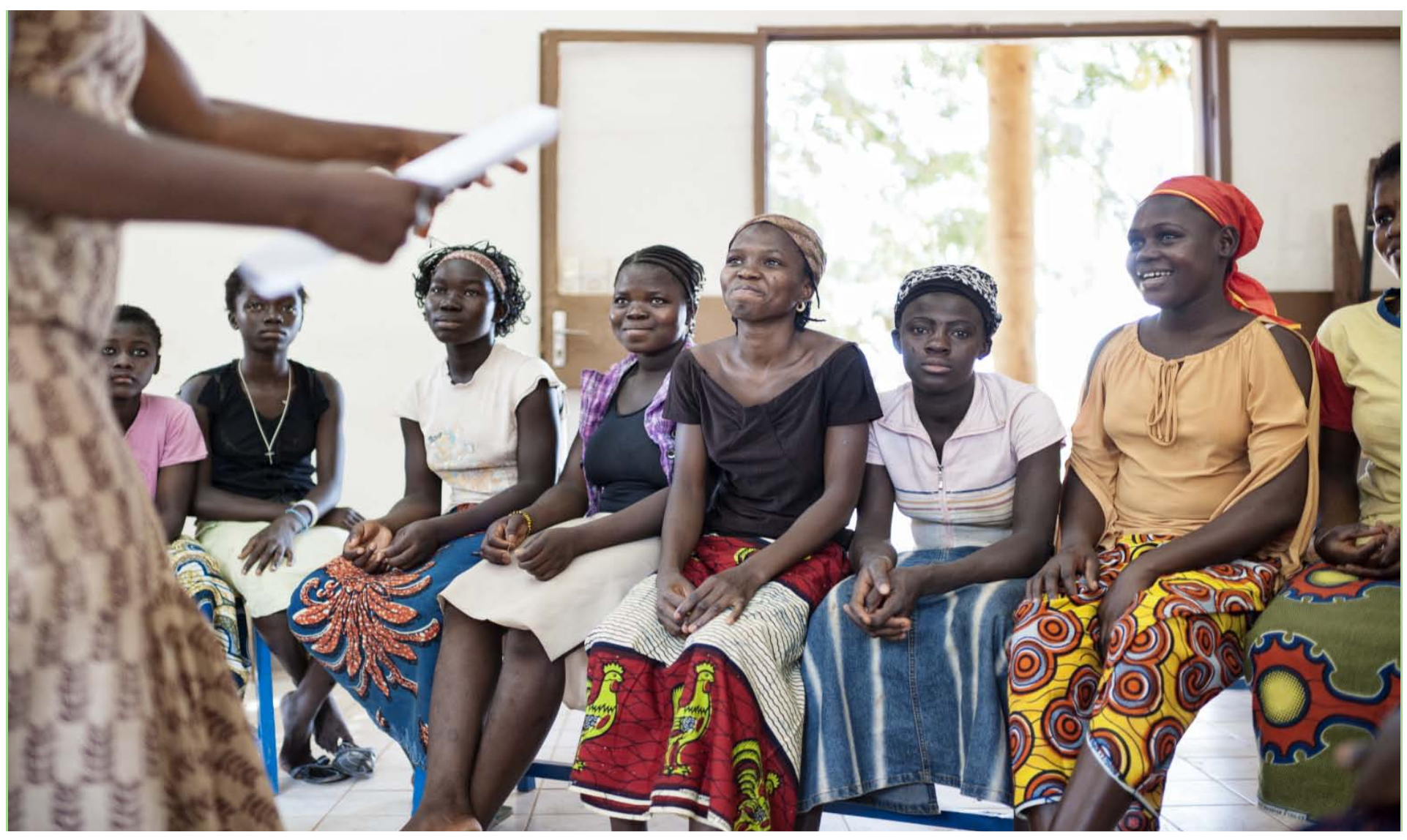




\title{
(2) Population Council
}

Le Population Council s'attelle aux questions cruciales de la santé et du développement - qu'il s'agisse d'endiguer la progression du VIH, d'améliorer la santé reproductive et d'offrir aux jeunes la promesse d'une vie satisfaisante et productive. Par ses travaux de recherche en biomédecine, en sciences sociales et en santé publique dans 50 pays, il s'efforce d'apporter avec ses partenaires des solutions qui conduisent à des politiques, des programmes et des technologies plus efficaces, pour améliorer la vie partout dans le monde. Fondé en 1952, le Population Council est une organisation non gouvernementale à but non lucratif. II siège à New York sous la conduite d'un conseil d'administration international.

\author{
Population Council \\ One Dag Hammarskjold Plaza \\ New York, NY 10017 \\ Population Council \\ 01 BP 6250 \\ Ouagadougou 01 \\ Burkina Faso
}

www.popcouncil.org

Indication de source suggérée: Sarah Engebretsen. 2012. "Observations initiales et finales de Filles éveillées : Programme pilote de renforcement des compétences des adolescentes migrantes employées de maison Cohorte 1 (2011-2012), Bobo-Dioulasso." Ouagadougou: Population Council.

Photo courtoisement offerte par Ollivier Girard

(C) 2012 The Population Council, Inc. 



\section{CONTENU}

Contexte.

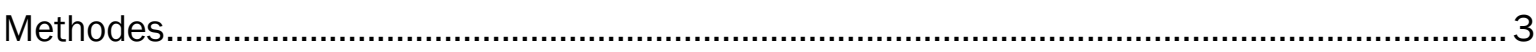

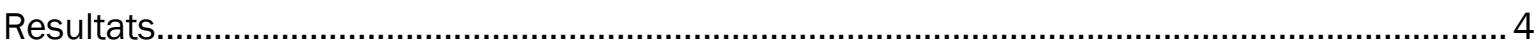

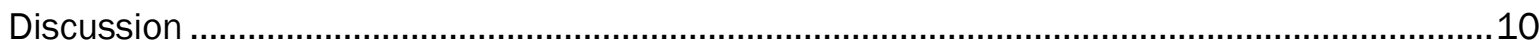


Peuplé à 45,2\% de jeunes de moins de 15 ans, le Burkina Faso abrite la 10e population la plus jeune du monde. ${ }^{1}$ Les jeunes Burkinabè n'ont guère de débouchés économiques, en particulier dans les milieux ruraux. Le problème concerne tout particulièrement les adolescentes, qui n'ont généralement bénéficié que d'une très faible scolarisation ou autre préparation à l'emploi. L'impasse économique alimente l'exode rural et la migration - interne aussi bien qu'internationale - vers les milieux urbains, à la recherche de travail. Pour beaucoup de filles, un emploi de maison urbain est l'occasion de gagner de l'argent, souvent dans le but d'acheter le trousseau nécessaire au mariage. Seules, les filles sont physiquement et économiquement vulnérables. Beaucoup courent un risque élevé de violence sexiste. Pressées de gagner leur premier salaire, elles acceptent parfois des conditions d'emploi dangereuses et mal rémunérées.

\section{Constats de la recherche formative}

Sous subvention de la Jacobs Foundation, le Population Council a mené en 2010 une recherche formative dans le but de mieux comprendre la situation des adolescentes migrantes employées de maison au Burkina urbain. L'étude a procédé par analyse de la documentation existante sur les adolescentes employées de maison, discussions de groupe avec ces adolescentes et entretiens en profondeur avec les employeurs, les logeurs et les organisations non gouvernementales qui interviennent auprès de ces adolescentes et/ou filles migrantes. II en ressort que les filles quittent leur foyer d'origine et migrent faute de débouchés. Arrivées à la ville, elles vivent généralement chez leurs employeurs et travaillent de longues journées laborieuses. II leur reste très peu de temps pour aller à l'école, développer des réseaux sociaux et acquérir les compétences nécessaires à la vie adulte.

Les résultats de la recherche indiquent également que les adolescentes migrent souvent par étapes - vers une petite ville dans un premier temps, puis vers la capitale. De plus, la migration des filles est généralement saisonnière : elles vivent et travaillent dans les villes durant la saison sèche et retournent chez elles chaque année pour aider aux travaux des champs pendant la saison des pluies. La recherche formative révèle que les programmes destinés à cette population interviennent en majorité après l'arrivée d'un malheur, plutôt que de développer et renforcer la capacité des filles à se protéger ; qu'ils cherchent d'abord à informer les filles de leurs droits plutôt que de mettre l'accent sur leurs compétences et qualifications; qu'ils ne sont pas conçus en fonction de segments d'âge logiques et qu'ils se concentrent sur les intrants (tels que le nombre de messages diffusés à la radio) plutôt que sur le changement mesurable au niveau de la fille.

\footnotetext{
1 Population Reference Bureau. (2011). World Population Data Sheet. Site consulté le 22 juillet 2012 sur http://www.prb.org/pdf11/2011population-data-sheet eng.pdf
} 


\section{Le programme Filles éveillées}

Face à ce bilan, le Population Council a conçu à l'intention des adolescentes migrantes employées de maison au Burkina urbain une intervention de 30 sessions menées en espaces sûrs sur un période de huit mois. Filles éveillées cherche à accroître le capital social des adolescentes, à renforcer leurs compétences sur le plan de la santé et de l'hygiène (y compris la santé sexuelle et reproductive), leurs compétences de vie courante et leurs capacités financières, et à établir le lien entre les filles et les services. En apportant aux filles des espaces sûrs, un accès à leurs pairs et l'assistance d'une femme mentor, ce programme entend ouvrir de nouveaux débouchés aux employées de maison et réduire leurs vulnérabilités. Le programme pilote 2011-2013 s'organise en deux cycles. La cohorte 2011-2012 a été menée à BoboDioulasso - plus spécifiquement dans les quartiers de Diarradougou dans le secteur 2 ; de Koko, entre les secteurs 4, 15 et 16 ; de Sikassocira dans le secteur 8; d'Accart-Ville, entre les secteurs 9 et 10 ; de Sarfalao dans le secteur 17 ; et de Lafiabougou dans le secteur 20. La cohorte 2012-2013 sera sélectionnée à Ouagadougou ainsi qu'à Bobo-Dioulasso.

Recrutement : Les mentors ont été recrutées dans les communautés de sélection après annonces faites par les dirigeants locaux et les crieurs publics. Les candidatures ont été soumises à l'organisation chargée de la mise en œuvre, l'Association Tié. Les candidates retenues ont été interviewées et les mentors et leurs remplaçantes éventuelles ont été sélectionnées par un comité de trois membres de l'organisation de mise en œuvre. Les mentors étaient des femmes bien connues et respectées de leur communauté. Âgées de 20 à 30 ans, elles savaient lire et écrire, parlaient la langue dioula et avaient atteint un niveau de scolarisation de quelques années secondaires au moins. Avant de procéder au recrutement de participantes, les mentors de Bobo ont reçu une formation de trois jours organisée par l'Association Tié. Cette formation a porté sur les objectifs du programme, les exigences relatives aux visites de maison, les réunions communautaires, les outils d'évaluation et une analyse détaillée de chaque leçon du programme. Après cette formation et les sessions ultérieures d'approche communautaire, les mentors se sont rendues de porte à porte dans les communautés sélectionnées afin d'identifier les filles admises à participer et de sensibiliser la communauté au programme Filles éveillées. Les filles migrantes de 11 à 16 ans $^{2}$ qui étaient employées de maison sur l'un des sites de projet sélectionnés ont été invitées à participer au programme. L'autorisation de leurs employeurs ou gardiens a été demandée. Les participantes et leurs employeurs ont été invités à signer le formulaire d'autorisation de participation au programme et le consentement à la participation des filles aux enquêtes afférentes. Au total, 180 filles ont été recrutées pour la première cohorte du programme.

Participation aux groupes de filles : Étant donné les contraintes de temps de la population ciblée, les groupes se sont réunis une fois par semaine, le dimanche, pendant environ deux heures. Les rencontres ont eu lieu dans les classes vides des écoles locales, des immeubles municipaux et

\footnotetext{
2 La tranche d'âge cible du programme est celle de 11 à 18 ans mais ce rapport ne concerne que les observations relatives à la Cohorte 1 de Bobo-Dioulasso, où les résultats de la recherche formative ont dicté des critères d'admissibilité de 11 à 16 ans. La cohorte 2012-2013 à Ouagadougou inclura des filles de 15 à 18 ans.
} 
des ONG locales, ainsi que dans une cour semi-fermée. Les sessions ont été conçues selon un modèle interactif avec discussions de groupe, récits personnels, exercices et jeux de rôle, dans le but d'apprendre aux filles des compétences spécifiques de vie courante, de santé et d'hygiène, de santé sexuelle et reproductive et d'éducation financière. Tous les groupes ont achevé les 30 sessions, même si certains ont dû le faire en moins de huit mois (voir plus loin la note 3 ). En moyenne, les participantes au programme Filles éveillées ont assisté à 22 des 30 sessions. Soixante-douze pour cent ont assisté à au moins 20 sessions et $20 \%$ à la totalité des 30 sessions.

Le programme Filles éveillées a été évalué selon le plan pré- et post-test, par évaluation des améliorations du capital social des participantes ainsi que de la connaissance, des attitudes et des comportements sur les points clés du programme. Ce rapport explore les observations initiales et finales (base et fin) relatives aux adolescentes de 11 à 16 ans ayant participé à la Cohorte 1 du programme Filles éveillées à Bobo-Dioulasso en 2011-2012.

\section{MÉTHODES}

\section{Conception des outils et collecte des données}

Les outils d'enquête de base et finale sont le produit d'une collaboration entre le personnel du bureau burkinabè et du siège du Population Council. Ils sont inspirés des questionnaires utilisés par le Population Council dans des enquêtes sur les adolescents menées auprès de populations similaires dans d'autres pays d'Afrique subsaharienne. Adaptées au contexte du Burkina, les questions ont aussi été affinées pour mieux cerner l'information spécifique aux filles migrantes et employées de maison. Les questions de l'enquête étaient en grande partie de type fermé. La collecte de données à porté sur les caractéristiques sociodémographiques, les compétences de vie et le capital social, la santé et l'hygiène, la santé sexuelle et reproductive, les capacités financières et le genre. L'enquête finale était pratiquement identique à celle de base initiale, si ce n'est que le questionnaire final comprenait aussi des questions sur les attitudes à l'égard du programme.

Le pré-test s'est déroulé début novembre 2011 à Diarradougou, Koko et Accart-Ville et début 2012 à Sikassocira, Sarfalao et Lafiabougou. ${ }^{3}$ Le programme Filles éveillées a débuté peu après l'accomplissement du pré-test. Une enquête post-test a été menée fin juin/début juillet 2012, après la conclusion du programme.

Étant donné la nature plutôt sensible des sujets abordés, les entretiens ont été menés strictement par des femmes, répondant aux critères de qualification minimum suivants : éducation universitaire ; maîtrise de la langue dioula, parlée localement à Bobo-Dioulasso ;

\footnotetext{
3 Selon la conception initiale, la Cohorte 1 aurait dû avoir lieu à Bobo-Dioulasso et Ouagadougou Cependant, des difficultés survenues au niveau d'un sous-bénéficiaire de Ouagadougou ont exigé la révision du plan avec recrutement de groupes additionnels de filles à Bobo-Dioulasso, ce qui explique la mise en œuvre retardée de certains groupes par rapport aux autres.
} 
expérience de la collecte de données quantitatives ; sensibilité aux questions relatives aux adolescentes vulnérables et au genre ; et capacité de travail en équipe. Le programme a fait appel à des intervieweuses relativement jeunes (24 à 26 ans) pour que les adolescentes se sentent à l'aise lors de la réponse aux questions. Les intervieweuses ont participé à une formation complète sur les objectifs du programme, les techniques d'entrevue importantes et les jeux de rôle, la traduction du questionnaire dans la langue locale, ${ }^{4}$ la nécessité d'un espace privé durant la collecte des données et l'importance de la confidentialité. Après cette formation, les intervieweuses de l'enquête de base ont pré-testé les questionnaires dans les quartiers avoisinants dans le but de clarifier les questions ou les catégories de réponse qui échappaient aux répondantes. Quelques légères modifications ont été apportées au questionnaire avant sa soumission à la population cible. Dans la mesure du possible, les intervieweuses de base ont été retenues pour la collecte des données finales, afin d'assurer l'efficacité du processus d'enquête finale.

La supervision de la collecte des données a été confiée à un consultant expérimenté au service de l'Institut Supérieur des Sciences de la Population à l'Université de Ouagadougou. Ce consultant a également facilité la formation des intervieweuses, la saisie des données, le nettoyage des données et le recodage des variables. Les entretiens ont été menés en privé, à l'écart des employeurs et autres membres du ménage, pour que les filles puissent répondre à l'aise aux questions. Les entretiens ont duré entre 45 minutes et une heure. Étant donné l'emploi du temps chargé des employées de maison, les intervieweuses ont dû faire preuve de souplesse dans la planification de chaque entretien au moment qui convenait le mieux à la fille.

Les questionnaires remplis ont été soumis à un contrôle de qualité des données et de complétude. Les données ont été saisies dans le logiciel d'enquête SPHINX, renommé pour sa capacité de contrôle des erreurs de collection de données. Les erreurs étant cependant inévitables, le nettoyage des données initiales et finales a été effectué sur une période de deux à trois semaines, avec tests de cohérence exécutés sous STATA et SPSS. Les données protégées par mot de passe ont été envoyées du bureau burkinabè du Population Council au siège de l'organisation, où elles ont été analysées sous SPSS 17 avec l'aide de l'équipe Burkina Faso.

\section{RÉSULTATS}

\section{Caractéristiques de l'échantillon}

Au total, 180 filles ont répondu à l'enquête initiale : 90 dans le cadre de la collecte de données de novembre 2011 à Diarradougou, Koko et Accart-Ville et 90 début 2012 à Sikassocira, Sarfalao et Lafiabougou. L'échantillon final compte un total de 129 filles, avec non-participation à l'enquête finale d'une part disproportionnée des filles de Sikassocira. Cette perte au suivi est

\footnotetext{
4 Les questionnaires, rédigés en français, ont été administrés en dioula. Dans les cas où les filles ne parlaient pas le dioula, le questionnaire a été administré en dagara.
} 
imputable à l'arrivée inhabituellement précoce de la saison des pluies 2012, cause du retour de nombreuses filles dans leurs villages pour aider aux travaux agricoles.

Sept des 180 répondantes à l'enquête initiale ont déclaré avoir dépassé la tranche d'âge cible de 11 à 16 ans et ont par conséquent été exclues de l'analyse de base et du programme. ${ }^{5}$

Environ un quart des répondantes ont été qualifiées de jeunes adolescentes (11 à 13 ans). Six pour cent ont déclaré ne pas savoir leur âge à la base, ce qui n'est pas rare dans la population cible. Ces filles ont été gardées dans l'analyse et le programme car elles avaient déclaré un âge compris dans la tranche cible au recrutement et semblaient répondre aux critères d'admissibilité. II convient de remarquer ici que près du tiers des filles ont déclaré à la base ne pas avoir de documents personnels tels qu'une carte d'identité faisant état de leur date de naissance. À l'enquête finale, 4\% seulement ont déclaré ne pas savoir leur âge, signe d'amélioration peut-être de la conscience de soi.

La population de l'échantillon est particulièrement mobile et la migration interne, plutôt qu'internationale, est beaucoup plus courante parmi les participantes à Filles éveillées. Quatrevingt-seize pour cent des participantes ont déclaré être nées au Burkina ; les autres étaient nées dans les pays voisins du Mali et de la Côte d'Ivoire. Lors de l'entretien initial, 61\% ont déclaré vivre dans leur quartier actuel depuis moins d'un an. Quatre cinquièmes des filles ont déclaré être venues à la ville pour raisons économiques et d'emploi. Plus de trois quarts vivaient avec leurs employeurs. Le plus haut niveau de scolarisation atteint et la connaissance du français se sont améliorés entre le pré- et le post-test (voir le Tableau 1), vraisemblablement sous l'effet de l'offre de participation facultative aux cours du soir, dont 59 filles ont profité dans la Cohorte 1.

TABLEAU 1 : Répartition (en \%) des filles interviewées du programme Filles éveillées en fonction de caractéristiques sélectionnées, suivant le moment de l'enquête.

\begin{tabular}{|l|l|l|}
\hline & Base (N=173) & Fin (N=129) \\
\hline Plus haut niveau de scolarisation atteint & & \\
\hline Primaire & $34 \%$ & $45 \%$ \\
\hline Secondaire & $3 \%$ & $4 \%$ \\
\hline Autre/hors système formel (système franco-arabe ou alphabétisation) & $6 \%$ & $5 \%$ \\
\hline Aucune scolarité & $57 \%$ & $46 \%$ \\
\hline & & \\
\hline Connaissance du français* & & \\
\hline Lit facilement & $8 \%$ & $16 \%$ \\
\hline Lit difficilement & $9 \%$ & $9 \%$ \\
\hline Ne sait pas lire & $83 \%$ & $74 \%$ \\
\hline
\end{tabular}

* La connaissance auto-déclarée a été confirmée en invitant les participantes à lire à haute voix cette simple phrase : "Je suis contente de participer au programme. "

\footnotetext{
${ }^{5}$ Les sept places ainsi libérées ont été ouvertes à d'autres filles pour atteindre un total de 180 participantes, bien que les données initiales des filles inscrites peu après le lancement officiel du programme ne soient pas disponibles.
} 


\section{Capital social et compétences de vie}

Filles éveillées a donné aux filles l'occasion d'étendre leurs réseaux sociaux en participant à des groupes de même sexe animés par des mentors féminines adultes. Les observations initiales indiquent que les adolescentes migrantes employées de maison sont fort isolées sur le plan social : 95\% ont déclaré ne faire partie d'aucun groupe social. À l'enquête finale, les filles n'ont déclaré aucune autre affiliation sociale (à un groupe de sport, de danse ou religieux, par exemple), laissant entendre que Filles éveillées était leur seule occasion d'affiliation formelle à une groupe. Des changements positifs sont observés, entre l'enquête initiale et finale, au niveau du capital social déclaré par les participantes, tel que mesuré par les réponses aux points "a un endroit sûr où rencontrer ses amies " (56\% vs $63 \%)$, "connaît quelqu'un à qui emprunter de l'argent en cas d'urgence " (62\% vs $72 \%$ ) et " connaît des personnes à qui parler et demander conseil " (91\% vs 98\%). À l'enquête finale, 97\% des filles ont convenu qu'elles avaient maintenant plus d'amies grâce au programme. Les mentors du programme faisaient aussi partie des réseaux sociaux étendus des participantes ; 100\% de l'échantillon final ont déclaré pouvoir parler à leurs mentors si un problème survenait dans leur vie. Outre l'expansion des réseaux sociaux, le programme a été conçu pour améliorer la capacité d'expression des filles : 99\% des répondantes à l'enquête finale ont déclaré qu'après leur participation au programme, elles se sentaient à l'aise pour résoudre leurs conflits avec autrui. Concernant l'objectif de renforcement de la confiance en soi, $96 \%$ des filles ont reconnu à l'enquête finale pouvoir s'exprimer avec plus de confiance depuis leur participation au programme. La Figure 1 présente les changements déclarés en termes de confiance en soi et de capacité d'expression personnelle.

FIGURE 1: Répartition (en \%) des filles interviewées du programme Filles éveillées en fonction des mesures de confiance en soi, suivant le moment de l'enquête.

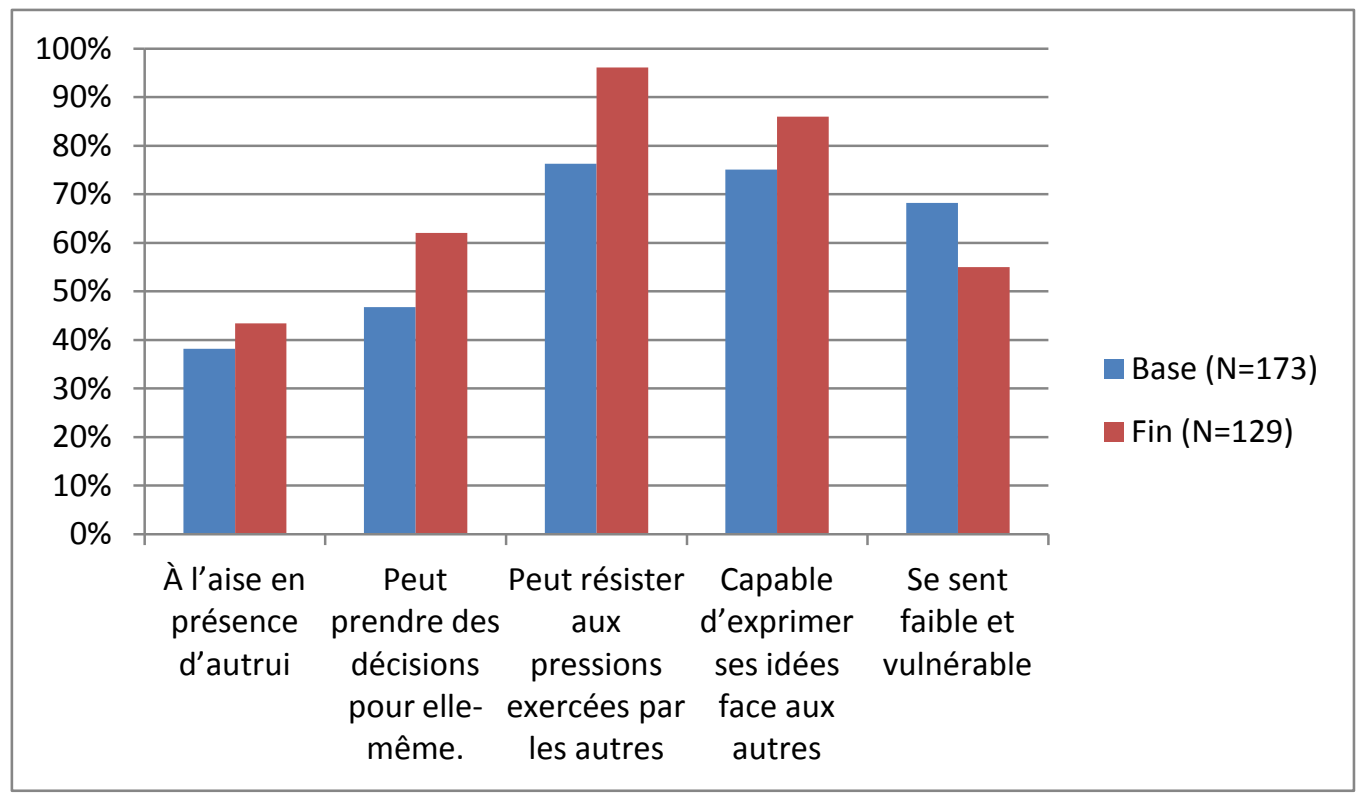




\section{Santé et hygiène}

Filles éveillées a donné à ses participantes l'occasion de s'informer sur d'importantes questions de santé et d'hygiène. Le programme a aussi apporté aux filles des compétences qui leur permettent de gérer leur propre santé et exploré les attitudes relatives aux comportements sains. Environ la moitié des filles ont déclaré se rendre dans un centre de santé en cas de maladie. Les raisons sinon citées sont : le manque d'argent, le sentiment de ne pas être suffisamment malade pour se faire soigner et les croyances que les centres de santé sont réservés exclusivement aux gens bien éduqués. À la question de savoir pourquoi certaines filles de leur âge se rendent dans les centres de santé, 12\% seulement des répondantes à l'enquête initiale savaient que ces centres peuvent fournir une information sur la santé. Ce pourcentage est cependant passé à $50 \%$ à l'enquête finale.

Les attitudes des filles à l'égard des centres de santé se sont améliorées avec le temps : à la base, $86 \%$ convenaient que les établissements de santé locaux étaient favorables aux filles, par rapport à $97 \%$ en fin de programme. Des changements positifs ont été observés aussi concernant la conscience des mesures à prendre pour améliorer sa santé personnelle : à la base, $10 \%$ seulement savaient qu'une fille pouvait s'auto-examiner les seins, par rapport à $54 \%$ à la fin. D'après les résultats du post-test, $99 \%$ des filles ont indiqué qu'elles pouvaient mieux gérer leur santé après leur participation à Filles éveillées.

La connaissance des filles en matière d'hygiène a été mesurée à travers des questions sur les circonstances dans lesquelles il convient de se laver les mains. Cette connaissance s'est avérée largement supérieure à celle attendue, 100\% de l'échantillon initial et final ayant répondu correctement à ces questions.

\section{Santé sexuelle et reproductive}

Compte tenu des observations de la recherche formative selon lesquelles les filles employées de maison ne sont guère informées sur les questions de santé sexuelle et reproductive et que leurs employeurs et logeurs ne se sentent pas aptes à leur en parler, le programme Filles éveillées les a instruites sur ces questions et sur les endroits où elles pouvaient accéder aux services offerts dans leurs communautés. La connaissance de certains aspects - notamment le fait qu'une femme peut tomber enceinte même après un seul acte sexuel non protégé, qu'une personne d'apparence saine peut être séropositive à VIH et la connaissance d'au moins une façon d'éviter le $\mathrm{VIH}$ - s'est révélée supérieure aux attentes initiales mais s'est aussi améliorée avec le temps, comme indiqué au Tableau 2 et aux Figures 2 et 3. De même, les attitudes favorables à l'égard du dépistage du VIH se sont révélées supérieures à celles attendues au pré-test, tout en s'améliorant toutefois entre la base et la fin (73\% vs $92 \%)$. 
TABLEAU 2 : Répartition (en \%) des filles interviewées du programme Filles éveillées en fonction de connaissances sélectionnées de santé sexuelle et reproductive, suivant le moment de l'enquête.

\begin{tabular}{|l|l|l|}
\hline & Base (N=173) & Fin (N=129) \\
\hline $\begin{array}{l}\text { Sait le moment du cycle menstruel où la femme peut tomber } \\
\text { enceinte }\end{array}$ & $1 \%$ & $35 \%$ \\
\hline $\begin{array}{l}\text { Sait qu'une femme peut tomber enceinte même après un seul acte } \\
\text { sexuel non protégé }\end{array}$ & $55 \%$ & $78 \%$ \\
\hline $\begin{array}{l}\text { Sait qu'une personne qui paraît en bonne santé peut être } \\
\text { séropositive à VIH }\end{array}$ & $67 \%$ & $88 \%$ \\
\hline Nombre de moyens corrects cités d'éviter le VIH : & $22 \%$ & $10 \%$ \\
0 & $62 \%$ & $48 \%$ \\
1 & $16 \%$ & $42 \%$ \\
\hline $2+$ & & \\
\hline
\end{tabular}

FIGURE 2: Répartition (en \%) des filles interviewées du programme Filles éveillées en fonction du nombre de méthodes de planification familiale identifiées, suivant le moment de l'enquête.

\section{Base}

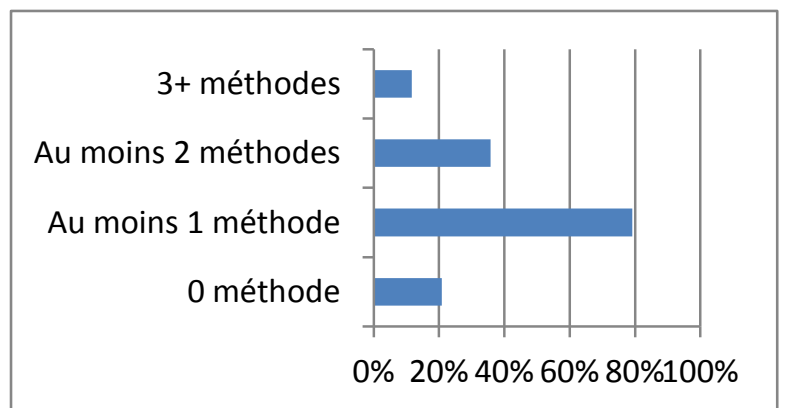

Fin

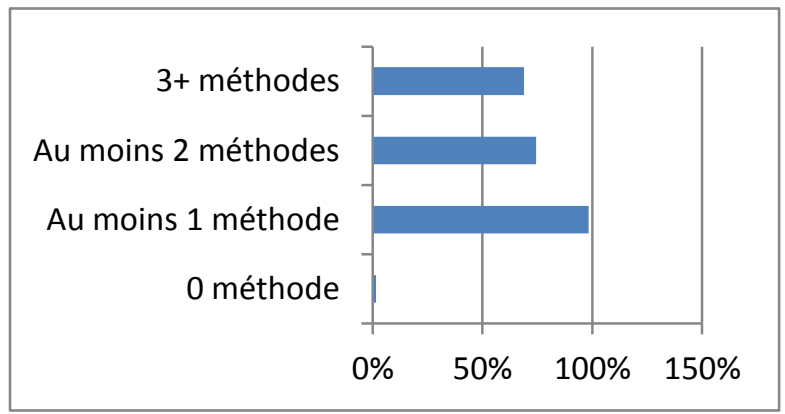

FIGURE 3 : Répartition (en \%) des filles interviewées du programme Filles éveillées en fonction du nombre de symptômes d'infection sexuellement transmissible identifiés, suivant le moment de l'enquête.

\section{Base}

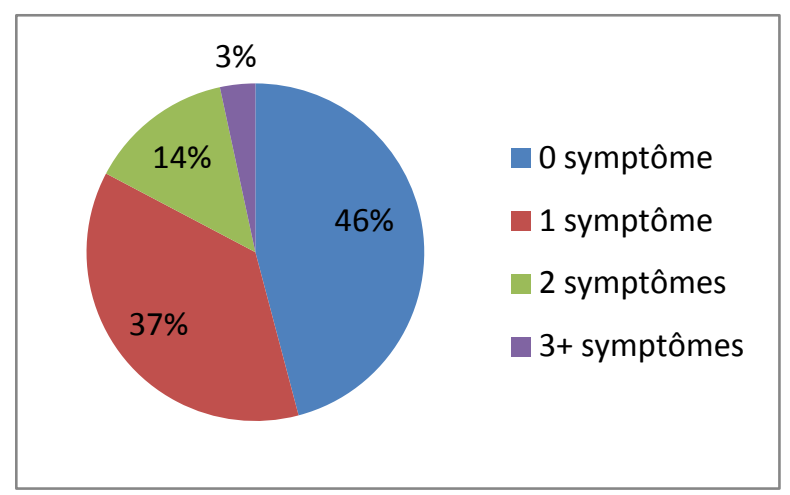

Fin

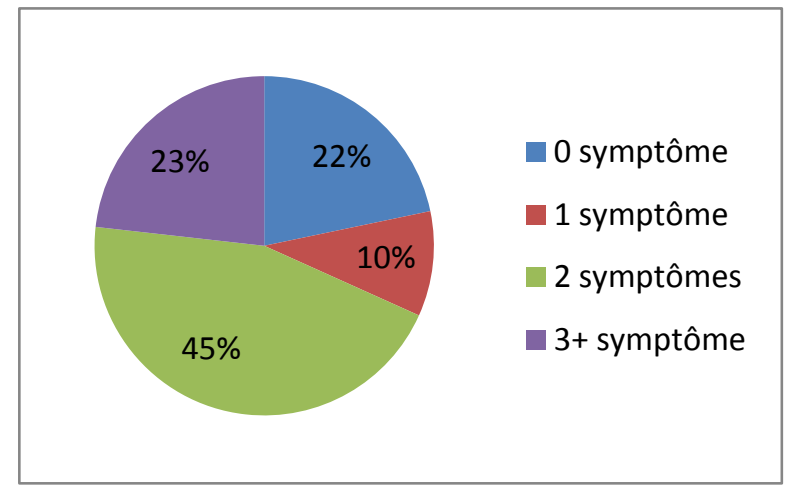


Outre l'amélioration de la connaissance, le programme a cherché à rendre les filles conscientes des services de santé disponibles dans leurs quartiers. La connaissance d'un endroit où se rendre dans la communauté pour le dépistage du VIH était élevée à la base mais s'est améliorée entre les pré- et post-tests ( $88 \%$ vs $100 \%$ ). La conscience d'au moins un endroit de la communauté où se procurer des méthodes de planification familiale est passée de $67 \%$ à la base à $91 \%$ à la fin. Celle d'un endroit où se rendre dans la communauté en cas de viol ou de harcèlement était de moins de $20 \%$ à la base mais s'est améliorée, passant à près de $50 \%$ à l'enquête finale.

\section{Capacités financières}

Les filles employées de maison sont déjà économiquement actives. Filles éveillées a renforcé leurs capacités d'épargne et de budgétisation, ainsi que leur aptitude à parler d'argent et à planifier leur avenir financier. En dépit du fait que plus de la moitié de ces filles n'avaient jamais fréquenté l'école avant de s'inscrire au programme, elles présentaient des capacités de calcul supérieures à celles attendues. À la base, 90\% ont répondu correctement à une question d'addition, l'explication en étant probablement le fait qu'elles échangent de l'argent avec les marchands lors de leurs achats pour le compte de leurs employeurs.

Les déclarations relatives aux principaux objets de dépense de leur argent restent constantes entre les enquêtes initiale et finale : il s'agit notamment de produits de beauté, de vêtements et d'envois d'argent à leur famille. À la question de savoir qui décide comment elles dépensent leur salaire, environ deux tiers des filles de l'échantillon déclarent prendre elles-mêmes ces décisions, avec une légère amélioration entre les observations de base et finales (65\% à 69\%). En fin de programme, les filles étaient plus susceptibles de déclarer avoir un plan de dépenses par rapport à la base (93\% vs $81 \%$ ) - résultat, probablement, de la session sur les capacités financières consacrée à ce sujet.

Les comportements d'épargne se sont améliorés de la base à la fin (81\% à 90\%). En fin de programme, les filles se sont aussi montrées plus susceptibles de déclarer faire des économies pour parer aux urgences ou aux problèmes qui risquent de survenir ( $56 \%$ vs $43 \%$ à la base). Les autres raisons notables d'économie incluent l'achat d'articles personnels et l'envoi d'argent aux familles. Les réponses à la question de savoir où elles gardent leurs économies sont diverses. On notera toutefois que le pourcentage déclarant les garder à la maison diminue de $24 \%$ à $14 \%$ entre le début et la fin du programme, tandis que celui des filles qui épargnent à la banque ou dans une tontine augmente de 3\% à 14\%. À l'enquête finale, 90\% des filles indiquent que le programme leur a appris comment épargner.

De même, les déclarations de buts d'épargne s'améliorent entre les deux enquêtes : de $46 \%$ à $54 \%$ pour les objectifs à court terme (achat de vêtements et de produits de beauté, notamment) et de $68 \%$ à $75 \%$ pour ceux à long terme (trousseau et préparation financière au mariage). La capacité déclarée des filles de parler d'argent avec leurs employeurs s'améliore aussi entre les deux enquêtes (49\% à $78 \%$ ). 
Genre

Bien que le programme n'ait pas été conçu dans le but de changer les attitudes ou les normes de genre, les pré- et post-tests ont évalué la question afin de déterminer si la participation à un groupe de renforcement de compétences strictement féminin avait un effet indirect sur les attitudes de genre au fil du temps. Du pré- au post-test, les attitudes des filles sont devenues plus équitables en termes de genre (Tableau 3), bien que le changement observé au fil du temps se révèle moins significatif pour certains indicateurs que pour d'autres. Par exemple, l'idée que la femme doit demander la permission à son mari change minimalement du début à la fin du programme. Le changement des perspectives de genre profondément ancrées nécessite des initiatives à long terme faisant entrer en jeu de multiples intervenants.

TABLEAU 3 : Répartition (en \%) des filles interviewées du programme Filles éveillées qui se disent d'accord avec les énoncés d'équité de genre, suivant le moment de l'enquête.

\begin{tabular}{|l|l|l|}
\hline & Base (N=173) & Fin (N=129) \\
\hline $\begin{array}{l}\text { Les garçons doivent passer la même quantité de temps } \\
\text { que les filles aux tâches ménagères }\end{array}$ & $22 \%$ & $40 \%$ \\
\hline $\begin{array}{l}\text { Les filles doivent pouvoir décider de la personne qu'elles } \\
\text { vont épouser }\end{array}$ & $84 \%$ & $94 \%$ \\
\hline $\begin{array}{l}\text { Les jeunes doivent être informés sur les questions de la } \\
\text { planification familiale, de la grossesse et de } \\
\text { l'accouchement avant de se marier }\end{array}$ & $89 \%$ & $99 \%$ \\
\hline $\begin{array}{l}\text { Les garçons doivent avoir la priorité sur les filles en ce qui } \\
\text { concerne la scolarisation }\end{array}$ & $54 \%$ & $43 \%$ \\
\hline $\begin{array}{l}\text { Le mari doit avoir le droit de battre sa femme si elle ne lui } \\
\text { obéit pas }\end{array}$ & $58 \%$ & $31 \%$ \\
\hline $\begin{array}{l}\text { La femme doit toujours demander la permission de son } \\
\text { mari avant de faire quoi que ce soit }\end{array}$ & $95 \%$ & $92 \%$ \\
\hline
\end{tabular}

*Les énoncés en italique sont considérés contraires à l'équité de genre.

\section{DISCUSSION}

Le programme Filles éveillées a été mis au point dans le but d'accroître les réseaux sociaux et de développer les compétences des adolescentes migrantes employées de maison au Burkina Faso urbain. Bien qu'il existe d'autres programmes au service de cette population, Filles éveillées est I'un des premiers à viser le renforcement des compétences de santé, sociales et économiques des filles avant que ne survienne un malheur dans leur vie. Les filles ont accueilli fort positivement le programme : $98 \%$ ont déclaré en fin d'intervention qu'elles pensaient désormais plus à leur avenir qu'avant leur participation. De même, toutes les filles de l'échantillon final ont déclaré qu'elles recommanderaient Filles éveillées à d'autres filles dans leur situation.

La première cohorte a montré que malgré leur emploi du temps chargé, les adolescentes migrantes s'intéressent au programme et peuvent assister à ses sessions hebdomadaires. Les 
résultats des pré- et post-tests de la Cohorte 1 révèlent une amélioration mesurable au niveau de la fille sur tous les points d'intérêt : réduction du pourcentage de filles qui n'avaient jamais été scolarisées (vraisemblablement grâce au soutien programmatique de l'éducation des filles intéressées), redoublement du pourcentage de filles ayant appris à lire, élargissement des réseaux sociaux et filets de sécurité des filles, amélioration des déclarations de confiance en soi, meilleure connaissance de la façon de gérer sa santé, meilleure connaissance de la santé sexuelle et reproductive (VIH compris), sensibilisation accrue aux services de santé disponibles dans son quartier, meilleur comportement d'épargne et buts d'épargne accrus, et soutien supérieur de normes de genre équitables (même si ce point n'est pas inclus dans les objectifs explicites du programme).

Les notes des intervieweuses concernant l'enquête finale font aussi état d'une amélioration notable, signe de visibilité du gain de connaissances des filles dans leur façon de répondre aux questions et de donner des réponses utiles plutôt que de choisir l'option "Je ne sais pas ". La confiance, l'estime de soi et le capital social sont les pierres angulaires de ce programme mais peuvent être difficiles à mesurer dans les enquêtes standard. Les notes d'entretien final des intervieweuses indiquent que, dans l'ensemble, les filles s'exprimaient sans embarras et avec plus de confiance par rapport à l'enquête initiale. Les observations des mentors et des chargés de programme de l'Association Tié sont similaires à tous les niveaux, laissant entendre l'efficacité du programme à aider les filles à s'exprimer plus librement et à développer leurs réseaux sociaux. Étant donné l'importance du capital social et des capacités financières dans l'aide aux filles vulnérables à surmonter les difficultés de leur vie, l'équipe du Population Council a décidé de compléter les observations initiales par la collecte de données qualitatives dans ces deux domaines particuliers. Cette collecte a été effectuée à la mi-2012 et ses résultats sont résumés dans un autre rapport.

En sa qualité de programme pilote, il était inévitable que Filles éveillées ne rencontre d'obstacles Iors de sa première itération. Cette première cohorte a permis de tester le modèle programmatique et les outils de collecte de données, en vue de leur amélioration. La planification de deux cohortes successives donne à l'équipe responsable de la recherche et du programme l'occasion et le temps de tester le modèle et les outils, de réfléchir aux résultats de la première cohorte et d'apporter les ajustements nécessaires avant le lancement de la seconde. Les observations quantitatives et celles de l'évaluation du processus (objet d'un rapport séparé) ont révélé la nécessité d'affiner la conception et la mise en œuvre du programme. Par exemple, les observations initiales semblent indiquer une bonne maîtrise de l'hygiène des mains parmi les filles, alors que les notes de session des mentors révèlent une faible connaissance de l'hygiène dentaire. Le programme d'enseignement a par conséquent été revu pour consacrer moins de temps à la question de se laver les mains et ajouter en revanche une session sur l'hygiène dentaire. De même, la nouvelle approche tient compte de l'estimation des mentors concernant la section sur la santé sexuelle et reproductive, qu'elles ont jugée trop dense. Le programme révisé simplifie l'information présentée sur l'appareil génital féminin et masculin, ainsi que les questions posées aux filles pour évaluer leur compréhension de cette information. II présente aussi un plus grand nombre d'images et d'histoires de filles individuelles. 
Cette étude présente plusieurs limites. D'abord, le plan d'étude est limité par l'absence d'un groupe témoin. Les changements observés chez les participantes entre les pré- et post-tests pourraient être dus à des facteurs indépendants de Filles éveillées. Les itérations futures du programme bénéficieraient d'un plan comparatif randomisé : les risques d'invalidité interne en seraient minimisés et un lien de causalité plus ferme pourrait être établi entre l'apport de Filles éveillées et les résultats observés.

Ensuite, le programme a été conçu pour coïncider avec la saison sèche, lorsque les filles migrantes vivent et travaillent en ville, et la collecte des données était censée avoir lieu juste avant le lancement du programme et juste après sa conclusion. Or la saison des pluies 2012 s'est déclarée un peu plus tôt que d'habitude, amenant de nombreuses filles migrantes à regagner leurs villages d'origine plus tôt que les autres années. Par conséquent, beaucoup de participantes ont quitté le programme avant sa conclusion officielle, manquant par conséquent aussi la collecte de données finales. Cet imprévu a eu pour effet une réduction de la taille d'échantillon, pour la collecte des données finales, par rapport à celui de la collecte initiale. II se peut que le programme en ait subi une perte sélective, avec biais de l'échantillon final dans le sens des migrantes mieux assimilées à la vie urbaine. La seconde cohorte, en 2012-2013, débutera et s'achèvera un peu plus tôt dans un effort de conservation d'autant de participantes que possible sur toute la durée du programme, avec minimisation des pertes avant la collecte de données finale.

Au-delà de la portée d'évaluation de programmes à faible échelle tels que Filles éveillées, une recherche ultérieure est nécessaire si l'on veut mieux comprendre le vécu des adolescentes migrantes fraîchement arrivées à la ville. Les enquêtes démographiques pourraient combler le vide, de par leur plus grande envergure, mais ni l'un ni l'autre des grands programmes internationaux actuels - les Enquêtes démographiques et de santé (EDS) menées sous l'égide d'USAID et les Enquêtes en grappes à indicateurs multiples (MICS) de I'UNICEF - n'a jamais fait de la collecte de données sur la migration une priorité. De fait, les enquêtes DHS ont abandonné récemment les deux questions sur la migration sinon posées dans leurs questionnaires depuis plus de 20 ans. Le programme MICS n'a pour sa part jamais collecté la moindre information sur la migration. De même, il serait bon de rationaliser les définitions de la migration d'une enquête à l'autre, en vue d'estimations plus précises de son envergure et d'une allocation de ressources mieux éclairée. Une personne ayant migré vers une ville particulière en provenance d'une origine comprise dans la même grande région administrative serait certainement considérée comme migrante dans les enquêtes EDS, mais pas nécessairement dans le cadre d'un recensement, suivant la manière dont les déplacements régionaux internes y sont traités. Les décideurs politiques désireux d'établir des programmes pour adolescentes sur de solides bases factuelles, en fonction de données empiriques appelées à informer la décision quant à l'échelle des ressources et aux régions hautement prioritaires vers lesquelles elles doivent être ciblées, doivent savoir que différents messages risquent d'émaner de différentes sources de données. 
Les recommandations d'élargissement de la base factuelle sur les adolescentes migrantes seront traitées dans un rapport à paraître sur la population cible. ${ }^{6}$

Les conclusions de cette évaluation indiquent qu'il est possible d'apporter un changement mesurable dans la vie des adolescentes migrantes employées de maison à travers des programmes factuels ciblés qui renforcent leur capital social et les aident à acquérir des compétences de santé, sociales et économiques dans un espace sûr sous la conduite d'une femme mentor qui leur inspire confiance.

${ }^{6}$ Temin, Miriam, Mark Montgomery, Sarah Engebretsen, and Kate Barker. À paraître. Girls On The Move: Adolescent Girls and Migration in the Developing World. A Girls Count Report. New York : Population Council. 